\title{
The Use of a Palliative Care Screening Tool in the Intensive Care Unit
}

\author{
Wendy H Hatchell* \\ Francis Marion University, USA
}

*Corresponding author: Wendy H Hatchell, Francis Marion University, USA.

Received Date: March 17, 2020

Published Date: March 26, 2020

\section{Abstract}

Background: Patients with an abrupt life altering event or chronic illness are admitted to the intensive care unit (ICU) and frequently are at the end of life (EOL). Most often, these patients have not established or discussed their quality of life wishes at the EOL with their families or healthcare providers. This creates uncertainty and stress for the patients' loved ones, and ethical issues of patient pain and suffering while enduring futile lifesaving interventions.

Purpose: Address unmet patient pain, suffering, and right to autonomy needs; while fulfilling nursing standards of the Code of Ethics, nonmaleficence, beneficence, and respect in order to provide, implement quality of care, and prevent healthcare provider moral distress at the EOL. Ethics.

Theoretical Framework: Orem Self Care, American Association of Critical Care Nurse Synergy Model, Self-determination Theory, Code of

Methods: Utilization of an ICU palliative care screening tool to identify unmet patient needs at the EOL in the ICU.

Result: A retrospective analysis of patients who died in the ICU equaled an average length of stay (LOS) of 8.14 days, which is greater than the national LOS of 3.6 days. Application of the screening tool identified many patients with multiple categories of unmet patient needs and ethical dilemmas.

Conclusion and Implications: Utilizing a dedicated ICU screening tool can identify and support the need to address holistic and ethical issues, prevent and relieve pain and suffering and promote quality of life at the EOL in the ICU.

Keywords: Palliative care; Critical care; EOL; Policy; Ethics

\section{Background}

It is not unusual for patients to come into the hospital with a long-term illness or an abrupt life altering event and are at the end of life (EOL). When a life altering event occurs or if there is a sudden or drastic decline in an acute or chronic illness, these patients will be admitted or transferred to an intensive care unit (ICU). Of these patients $20 \%$ will die, most often due to advanced age or progression of an acute or chronic illness [1,2]. Additionally, those patients with chronic health conditions are living longer than ever, due to the advances in medicine. It is projected that $90 \%$ of patients aged 65 years and older have at least one chronic disease or condition, and at least $77 \%$ have two or more chronic diseases. It is further estimated by this year 2020, 157 million people will have a chronic illness [3]. With advances in healthcare, there is an expected increase in healthcare cost. ICU health care costs account for $\$ 82$ billion in the United States, which is $13 \%$ of inpatient hospital costs [4]. Of these ICU expenditures, $25 \%$ is spent on those who will die in the ICU [4]. These costs are related to the intensity of care and interventions used to sustain life in the ICU. In an ICU or critical care environment, interventions may involve lifesaving devices and procedures of invasive lines, being maintained on high maintenance intravenous (IV) pharmacologic medications, endotracheal tubes, and ventilators, which creates an inability to communicate and lack of autonomy for the patient. With these lifesaving devices, patients are in the ICU for an average of 3.8 days or longer [5]. 
Of those ICU patients who do survive hospitalization and are discharged, there is a $75 \%$ probability they will be readmitted to the hospital within 30 days or to a rehabilitation facility [6]. If these patients are readmitted within 30 days of discharge this becomes a non-reimbursable event for the organization, per regulations established by the Centers of Medicare and Medicaid Services, resulting in lost revenue [7,8]. Attempts are made to wean the patient from these lifesaving treatments when appropriate, but often to no avail. Most often this is the time when conversations are started with families regarding the futility of further treatment and goals of care. Unfortunately, the majority of these patients have not had a conversation with their family members and primary care providers regarding their wishes of medical treatments and quality of life when they are admitted to the hospital and are at EOL [9]. This situation arises due to the primary healthcare providers (HCP) lack of time to discuss the topic fully, as well as discomfort in discussing the topic. From the patient perspective, patients do not realize the significance of establishing these conversations early on in their life [9]. Many patients feel they have time to do this later or are uncomfortable with the subject [10]. They also may feel the HCP will bring the subject up when it is the appropriate time [9]. However, due to the lack of discussion regarding advanced care planning early on, these discussions typically occur with the patient's family when a crisis event occurs. This creates stress for loved ones, who may not know what their loved one would want, as well as not being prepared for what life-sustaining activities involve [9]. Many families have no concept of the seriousness of the events which are occurring and how it is affecting their loved one [9]. These conversations produce emotional, physical, and financial stress and burdens on family members, as well as inconsistencies with patient wishes if they were expressed and lack of patient comfort at the EOL [11]. It further creates moral and ethical distress for the nurses who are providing care for the patient.

Nurses must follow ethical principles of autonomy (patient's right to self-determination), nonmaleficence (obligation to do no harm), beneficence (obligation to do good), justice (fairness in treatment), fidelity (faithfulness), veracity (truthfulness), respect and the American Association of Nurses Code of Ethics with Interpretative Statements [12]. The Code of Ethics states nurses are to "...prevent and alleviate the cascade of symptoms and suffering that are commonly associated with dying" ("Code of Ethics"). To ease this burden, evidence-based interventions are needed to establish crucial conversations with patients, families or significant others in establishing goals of care with HCP's regarding their loved one's quality of life. An evidence-based intervention which "...bridges the gap between comfort and cure" [13] is palliative care. Not only is palliative care recognized as a crucial component of chronic disease, symptom and progression management, it is also a component of addressing the comprehensive care needs of critically ill ICU patients [6]. These needs include preventive and elimination of patient suffering, identification, and treatment of pain, symptoms, issues, and holistic needs [13]. Identification of these needs begins on admission in conjunction with coordination of empathetic and timely communication which focuses on addressing the patient's condition, prognosis and values, and families' needs and concerns. These factors should align to intensive care treatment goals and care transition planning [14]. The use of palliative care not only addresses the identification and needs of the critically ill patient but decreases hospital costs by decreasing LOS and utilization of resources [13]. Palliative care is further recognized by the World Health Organization (WHO), as an avenue to prevent and alleviate pain and suffering, as well as improve quality of life from problems arising from life threatening, as well as chronic illness, through early recognition and treatment [15]. The Institute of Medicine (IOM) also emphasizes the importance of establishing palliative care initiatives in "Dying in America: Improving Quality and Honoring Individual Preferences at the End of Life" [4]. These initiatives stress the importance of not only meeting patient needs, but also the need to provide and promote consistency with patient values and preferences of quality of life, and goals of care in the ICU at the EOL [4]. Additional organizations which support palliative care and its initiatives are the National Institute of Health (NIH), Robert Wood Johnson Foundation, American Association of Critical Care Nurses, American College of Critical Care Medicine, American Heart Association, American College of Cardiology Foundation, American Thoracic Society, American College of Chest Physicians, and others [14]. Currently, at a southeastern community hospital, patients are admitted into the ICU with a sudden, severe illness or a chronic condition. Presently, there is not a palliative care team at this community hospital to facilitate this goal of care conversation. Often families are reluctant to talk with nurses regarding EOL, or frankly do not want to discuss a non-curative option. Conversations regarding EOL are physician initiated, often several days after deterioration has started creating patient suffering; thus, making it imperative to establish a policy and/or program at this organization.

\section{Purpose}

The objective of this policy project was to utilize a palliative care screening tool to determine if a palliative care program would be of benefit at a southeastern community hospital.

\section{Methods}

\section{Evidence review and synthesis}

A literature review was conducted through PubMed, the Cumulative Index to Nursing and Allied Health Literature, and Scopus. The resulting studies utilized in the evidence review included systematic review and a retrospective study. The systematic reviews demonstrated a decrease in length of stay (LOS), ICU admissions, and ICU admission time to palliative care consult with the use of palliative care screening tools, as well as a decrease in family distress and anxiety while simultaneously improving communication through 'goals of care' conversations between family and care providers and improving quality of care $[14,16,17]$. May, Normand, and Morrison16 further identified 
that palliative care teams increased quality of care and decreased cost of care. Further studies by Jenko et al. [1] and Daly \& Matzel [3], substantiate the implementation of palliative care teams and palliative care screening in the ICU. Jenko et al. [1] study resulted in a $110 \%$ increase in palliative care referrals through utilizing a palliative care screening tool. The retrospective study by Lapp \& Iverson [2] identified the utilization of the Center to Advance Palliative Care (CAPC) ICU screening tool was more specific for identifying palliative care needs of the ICU patient than other available screening tools.

\section{Framework}

The optimal framework to implement this policy project was the IOWA model [18]. This model utilizes a logical step process. The first step address the state of current practice and the need for change. For this project collection of data consisted of obtaining ICU admission diagnosis, LOS, time of initiation of EOL communication with families, time to decision, and final disposition upon discharge from the ICU. The second step was to identify the key players, as well as barriers, with a dedicated interest in the initiation of palliative care screening tool within the ICU (nursing, HCP staff, leadership, chaplain, and social worker) who will advocate for the process and speak with leadership and stakeholders. Step three involved a review and analysis of the literature in seeking evidencebased solutions. The solution identified from the literature was to utilize a screening tool to capture unmet palliative care needs and determine if this information would substantiate need to develop initiate a palliative care team to address these needs and others $[1,2,19]$.

\section{Policy question}

Will an evidence-based ICU palliative care screening tool policy facilitate identification of patients who require palliative vs. curative care upon admission to the ICU at a southeastern community hospital?

\section{Project design}

The goal of this project was to identify unmet palliative care needs of those patients admitted to the ICU and who subsequently died in the ICU. The CAPC ICU screening tool was utilized to categorize historical data of those patients who met criteria of need for palliative care (Lapp \& Iverson, 2015). This project incorporated historical data of current practice obtained from a 3 month electronic medical health record (EHR) retrieval on diagnosis, LOS, course of events, initial physician conversation regarding the EOL, if and when families make EOL decisions, time to decision from initial physician conversation of EOL, and final disposition, from the previous year of 2017. The historical data was used to substantiate and support the need to implement a screening tool, evaluate patient palliative care needs, provide information of the need to establish a palliative care team and initiate a palliative care team consult.

\section{Result}

\section{Historical data}

Information was obtained from the EHR during January to March 2017 of those patients who died in the ICU (equaling 41 patients). The ages ranged from 33 to 100 years with the average age being 75 years. Average LOS equaled 8.14 days, which is greater than the national LOS of 3.6 days (Society of Critical Care Medicine, 2018). Admitting diagnoses were: abdominal pain (2), altered mental status (2), intracranial hemorrhage (1), end stage renal disease (1), seizures (1), cancer (3), chronic obstructive pulmonary disease (COPD) (3), chest pain (6), sepsis (6), respiratory failure (7), and cardiac arrest (9). The average time from admission to first discussion ranged from date of admission to 25 days, with an average of 3 days. Time from first discussion to decision ranged from 1 hour to 10 days depending upon family dynamics, with an average decision time of 61.4 hours. Historical data was placed on the CAPC ICU screening tool to determine who met admission criteria for meeting palliative care needs. Utilizing disease criteria: advanced stage IV cancer (6), multi organ failure (11), major acute neurologic insult (11), advanced dementia or other severe cognitive impairment (2), intracranial hemorrhage requiring mechanical ventilation (1), chronic renal disease or dialysis (4), post cardiac arrest (22), advanced COPD (9), and severe congestive heart failure (1). Utilization criteria results were: frequent hospital or ICU admission (5), greater than 1 ICU admission during the same hospitalization (1), admission from nursing home (6), and consideration to start renal replacement therapy (2). Other criteria results were conflicts regarding goals (9), and lack of social support (4) [2].

\section{Discussion}

\section{Summary}

Resulting analysis of the historical data revealed that CAPC Palliative Care ICU screening tool identified and supported the need to initiate and implement a palliative care policy to address holistic needs, prevent pain and suffering, and promote quality of life for patients and their families. The majority of the patients met multiple criteria of the screening tool; signifying the need for palliative care interventions. By initiating this needed change at admission, the LOS may be reduced, saving the hospital and family healthcare cost.

\section{Limitations}

Limitations were organizational buy out of the institution resulting in reappraisal of services provided.

\section{Implications/Conclusion}

Utilization of the ICU screening tool identified and supported the need to initiate and implement a palliative care policy to address holistic needs, prevent pain and suffering, and promote quality of life for patients who are at risk of dying in the ICU at 
this southeastern organization. This information supports that identification, initiation, and implementation of a dedicated palliative care screening tool can eliminate pain and suffering for patients by addressing personal and holistic preferences and needs. Despite issues with or the length of time it may take to go through an organizations policy process, one must be persistent and cognizant of the steps necessary to achieve needed evidencebased practice and change. It is important to remain focused on the goal and seek avenues in overcoming any obstacle. This change is needed to provide and improve patient outcomes. The cost of a palliative care initiative is costly upfront. However, the cost saved from reducing the LOS will balance the upfront cost.

\section{Acknowledgment}

None.

\section{Conflict of Interest}

No conflict of interest.

\section{References}

1. Jenko M, Adams JA, Johnson CM, Thompson JA, Bailey DE (2015) Facilitating palliative care referrals in the intensive care unit. Dimensions of Critical Care Nursing 34: 329-339.

2. Lapp EA, Iverson L (2015) Examination of a palliative care screening tool in intensive care unit patients. Journal of Hospice and Palliative Nursing 17: 566-574.

3. Daly D, Matzel S. (2013) Building a transdisciplinary approach to palliative care in an acute care setting. Journal of Death \& Dying 67: 4351.

4. Khandelwal N, Curtis JR (2014) Economic implications of end- of-life care in the ICU. Current Opinion Critical Care 20: 656-661.

5. Society of Critical Care Medicine (2018) Critical care statistics [Fact sheet].

6. Aslakson RA, Curtis JR, Nelson JE (2014) The changing role of palliative care in the ICU. Critical Care Medicine 42: 2418-2428.
7. Emanuel EJ (2013) Better if not cheaper. New York Times, USA, p.A23.

8. Cassel JB, Kerr KM, Kalman NS, Smith TJ (2015) The business case for palliative care: Translating research into program development in the U.S. Journal of Pain and Symptom Management 50: 741-749.

9. Houben CH, Spruit MA, Groenen MT, Wouters EF, Janssen DJ (2014) Efficacy of advance care planning: A systematic review and metaanalysis. Journal of the American Medical Directors Association 15: 477489.

10. Toraya C (2014) Evaluation of advance directives video education for patients. Journal of Palliative Medicine 17: 942-946.

11. Kross EK, Nielson EL, Curtis JR, Engelberg RA (2012) Survey burden for family members surveyed about end-of-life care in the intensive care unit. Journal of Pain and Symptom Management 44: 671-680.

12. Coyne PJ, Bobb B, Plakovic K (2017) Conversations in palliative care: Questions and answers with the experts (4th ed.) Pittsburg, PA: Hospice and Palliative Nurses Association.

13. Baker M, Luce J, Bosslet GT (2015) Integration of palliative care services in the intensive care unit: A roadmap for overcoming barriers. Clinical Chest Medicine 36: 441-448.

14. Asiakson R, Cheng J, Vollenweider D, Galusca D, Smith TJ, et al. (2014) Evidence-based palliative care in the intensive care unit: A systematic review of interventions. Journal of Palliative Medicine 17: 219-235.

15. World Health Organization (ND) Definition of palliative care [Fact sheet].

16. Khandelwal N, Kross EK, Engelberg RA, Coe NB, Long AC, et al. (2015) Estimating the effect of palliative care interventions and advance care planning on ICU utilization: A systematic review. Journal Critical Care Medicine 43: 1102-1111.

17. Nelson JE, Curtis R, Mulkerin C, Campbell M, Lustbader DR, et al. (2013) Choosing and using screening criteria for palliative care consultation in the ICU: A report from the improving palliative care in the ICU (IPALICU) advisory board. Critical Care Medicine Journal 41: 2318-2328.

18. Titler MG, Kleiber C, Steelman VJ, Rakel BA, Budreau G, et al. (2001) The IOWA model of evidence-based practice to promote quality care. Critical Care Nursing Clinics of North America 13(4): 497-509.

19. American Nurses Association (2015) In: The code of ethics for nurses with interpretive statements. Silver Spring, MD: Nursebooks.org 